\title{
Accelerated hepatocellular carcinoma recurrence rate after postoperative direct-acting antivirals treatment - preliminary report
}

\author{
Karola Warzyszyńska', Maurycy Jonas², Dariusz Wasiak ${ }^{3}$, Maciej Kosieradzki², Piotr Małkowski \\ ${ }^{1}$ Medical University of Warsaw, Poland \\ 2Department of General and Transplantation Surgery, Medical University of Warsaw, Poland \\ ${ }^{3}$ Department of Surgical and Transplant Nursing and Extracorporeal Treatment, Medical University of Warsaw, Poland
}

\begin{abstract}
Aim of the study: New interferon-free direct-acting antiviral (DAA) therapy has led to major progress in hepatitis C virus (HCV) treatment. Current outcomes are promising, especially in compensated cirrhosis. However, there are reports of accelerated hepatocellular carcinoma $(\mathrm{HCC})$ recurrence after surgery in patients treated with DAAs. The influence of DAA therapy on the timing and frequency of recurrence after surgical treatment needs further observation.

Material and methods: Fifty-one HCV infected patients with advanced liver cirrhosis and history of surgical treatment for HCC in 2012-2016 were analyzed in a case-control study. Nineteen patients received DAA therapy (DAA group) after tumor remission achieved by surgery and 32 patients were not treated with DAA (NDAA group). Follow-up included multiphase computed tomography scan or magnetic resonance imaging of the liver and alpha-fetoprotein level in 3-6-month intervals.

Results: An sustained virological response was achieved in 18 (95\%) DAA treated patients. Hepatocellular carcinoma recurrence was observed in $8(42.1 \%)$ patients from the DAA group and in 21 (65.6\%) from the NDAA group $(p=0.058)$. Relapse occurred within 265 days after surgery in the DAA group vs. 532 days in the NDAA group ( $p=0.033$ ). The one-year recurrence-free survival (RFS) rate was $47.3 \%$ vs. $75 \%$ in the DAA and NDAA group respectively $(p=0.45)$.

Conclusions: Use of DAA therapy in patients with a history of $\mathrm{HCC}$ may result in significantly accelerated relapse of the disease. The number of analyzed patients in this study is too small to state unquestionable conclusions. Further observation with a longer follow-up and larger patient group is needed. The study confirms that contemporary HCV treatment is highly effective.
\end{abstract}

Key words: hepatocellular carcinoma, direct acting antiviral drug, hepatitis $\mathrm{C}$ virus (HCV).

Address for correspondence

Karola Warzyszyńska, Medical University of Warsaw, 59 Nowogrodzka St., 00-001 Warsaw, Poland, e-mail: karola.warzyszynska@gmail.com

\section{Introduction}

Hepatitis C virus (HCV) infection leads to chronic liver inflammation that results in cirrhosis in $20-30 \%$ of patients $[1,2]$. Cirrhosis is the main risk factor for hepatocellular carcinoma (HCC), which occurs in $3.5 \%$ of these patients per year $[3,4]$. Nearly $44 \%$ of $\mathrm{HCC}$ cases in Poland are caused by HCV cirrhosis [5].
Over the last few decades, HCV treatment has consisted of pegylated interferon (PegIFN) combined with ribavirin. The outcomes were evaluated as sustained virological response (SVR) - no viral RNA detected in blood in 12 weeks after completed therapy, which was observed in only $50 \%$ of patient $[6,7]$. A positive response was correlated with significantly reduced risk of HCC occurrence [8-10]. 
Table 1. Patient groups characterization for tumor risk factors and time to direct-acting antiviral agents treatment introduction

\begin{tabular}{|c|c|c|}
\hline Factors & Direct-acting antiviral agents & Not treated with direct-acting antiviral agents \\
\hline Number of patients & $19(37 \%)$ & $32(63 \%)$ \\
\hline Number of SVR & $18(94.7 \%)$ & - \\
\hline Diameter of the tumor [mm] & $25.9(12-46)$ & $29.6(16-50)$ \\
\hline AFP before treatment $[\mathrm{mg} / \mathrm{dl}]$ & $139.2(2-880)$ & $32.4(1.7-198)$ \\
\hline AFP after treatment [mg/dl] & $19.2(0-122)$ & $11.6(0-28)$ \\
\hline \multirow[t]{2}{*}{ Time from surgery to DAA [days] } & $326.7(20-1513)$ & - \\
\hline & $\begin{array}{cc}\text { HCC recurrence (8 cases) } & \text { No HCC recurrence }(11 \text { cases }) \\
259.6(20-762) & 375.5(31-1531)\end{array}$ & \\
\hline
\end{tabular}

SVR - sustained virological response, AFP - alpha-fetoprotein, HCC - hepatocellular carcinoma, DAA - direct-acting antiviral agent

The recent introduction of the new, interferon-free direct-acting antiviral agents (DAAs) led to major progress in $\mathrm{HCV}$ treatment. According to the latest reports, an SVR is achieved in more than $90 \%$ of cases including both groups of patients, with compensated and decompensated cirrhosis (95-97\% vs. $85-90 \%$ respectively) [11-13]. Moreover, fewer contraindications and side effects compared to former therapy are noted [14]. Therefore, it is hoped that DAAs will provide better outcomes in prevention and treatment for both hepatic and extrahepatic HCV complications including HCC. However, there are reports of accelerated HCC recurrence after surgery in patients treated with DAA $[15,16]$. The influence of DAA therapy on timing and frequency of the recurrence after surgery needs further observation.

\section{Material and methods}

Fifty-one HCV infected patients with advanced liver cirrhosis and history of surgical treatment for HCC in the Department of General and Transplantation Surgery of Medical University of Warsaw in the years 2012-2016 were analyzed in a case-control study.

The group consists of 33 male and 18 female patients at the mean age of 58.5 (36-67), body mass index (BMI) of 26.1 (22.5-28.4) and tumor diameter of $27.5 \mathrm{~mm}$ (12-50 mm). Diabetes was observed in $44.4 \%$ of patients.

Nineteen patients received DAA therapy (DAA group) after tumor remission achieved by surgery and 32 patients were not treated with DAA (NDAA group). Both groups were comparable in terms of age, sex, BMI, progress of liver cirrhosis (Child-Pugh A or B), diameter and number of tumor foci and class $(0$ or $A)$ in the BCLC criteria. Initial alpha-fetoprotein (AFP) serum level was used only in the post-operative follow-up and it was not considered in the analysis due to weak predictive value. Surgical procedures included percu- taneous radiofrequency ablation (RFA), RFA at open laparotomy or non-anatomic liver resection. All these methods for HCC treatment were considered as equivalent, referring to the meta-analysis by Yi et al. [17] (Table 1).

Follow-up included multiphase computed tomography scan or magnetic resonance imaging of the liver and AFP serum level in 3-6-month intervals. The observation of any lesion with a characteristic HCC-like washout image in radiological examination was the basis to recognize the tumor relapse. Mean time from surgery to relapse was calculated for both groups to assess whether the tumor had recurred earlier in the DAA group. Moreover, one-year recurrence-free survival (RFS) rate, defined as the time from remission to any recurrence or any death according to the STEEP System proposed by American Society of Clinical Oncology (ASCO) [18], was estimated to unify the prognosis evaluation.

The whole group is under constant observation in our clinic. Patients not treated throughout one year are still without DAA therapy, although the majority of them are being qualified for the treatment program. The further results will be updated.

The quantitative variables are expressed as mean with the range in parentheses, and qualitative variables are expressed as absolute numbers with the percentages in parentheses. The $\chi^{2}$ test was used to determine whether there was a significant association between the study and control group. Kaplan-Meier estimates of the recurrence-free survival rates were calculated and compared using the Wilcoxon test. A $p$-value $<0.05$ was considered as statistically significant.

\section{Results}

An SVR was achieved in 18 (95\%) DAA treated patients. Hepatocellular carcinoma recurrence was observed in $8(42.1 \%)$ patients from the DAA group 


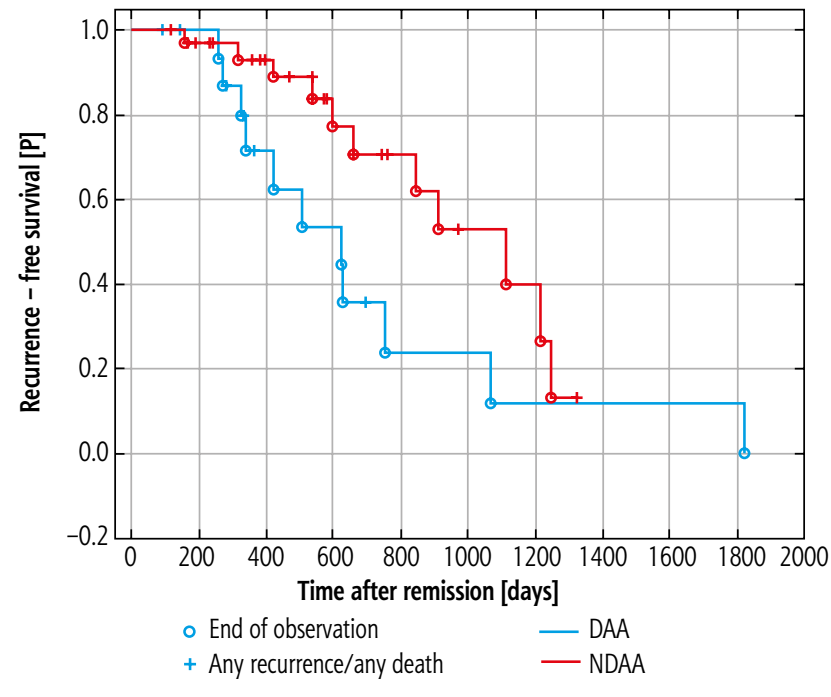

$D A A$ - direct-acting antiviral agents, NDAA - not treated with direct-acting antiviral agents

Fig. 1. Kaplan-Meier plot to compare hepatocellular carcinoma recurrence-free survival in both groups

and in $21(65.6 \%)$ from the NDAA group ( $p=0.058)$. There is no statistical evidence that the use of DAAs is associated with an increased rate of HCC recurrence. Relapse occurred within 265 (94-698) days after surgery in the DAA group vs. 532 (118-1360) days in the NDAA group, which was statistically significant $(p=0.033)$. The one-year recurrence-free survival (RFS) rate was $47.3 \%$ ( 9 patients) vs. $75 \%$ (24 patients) in the DAA and NDAA groups, respectively $(p=0.45)$ (Fig. 1).

\section{Discussion}

The new DAA therapy is certainly a breakthrough in HCV treatment. Effectiveness defined as an SVR is achieved in more than $90 \%$ of cases [11-13] in comparison to $50 \%$ in traditional PegIFN and ribavirin treatment $[6,7]$. Direct-acting antiviral agents also improve the function of the cirrhotic liver with a decrease of aminotransferase level in serum and reduction of fibrosis stage in FibroScan imaging. Moreover, many authors have stated that the use of contemporary treatment in patients with $\mathrm{HCV}$ reduces the risk of HCC incidence $[8-11,13]$ as well as IFN therapy [8-10].

However, some disturbing reports of an increased HCC recurrence rate after the previous surgery have emerged. An unexpected increase of the tumor relapse was noted by Conti et al. [16]. Another trial did not confirm a similar dependency [15], although there is a lack of convincing evidence to certainly prove it. Our study reveals a relevant difference in the HCC recurrence rate in DAA and NDAA groups (42.1\% vs. $65.6 \%$ respectively) although statistical significance was not achieved $(p>0.05)$. Meanwhile, some reports suggest possible acceleration of tumor relapse in the group of DAA-treated patients. Although the final recurrence rate is close to the result in the control group, it seems that the neoplasm may recur earlier in the postoperative period and spread more aggressively than the primal lesion $[15,16,20]$. In our trial recurrence was observed significantly earlier in the DAA than in the NDAA group (256 vs. 532 days after surgery, $p<0.05$ ).

Early recurrence may be correlated with numerous factors, such as diameter and volume of the tumor, cancer malignancy, number of lesions, invasion of the vessels, elevated level of AFP and progress of liver cirrhosis. In the material, the mean diameter of the tumor in the NDAA group was larger than in the DAA group (25.9 mm vs. $29.6 \mathrm{~mm}$ ), which could be the reason for the higher recurrence rate in the NDAA group. Meanwhile, the 4-times higher level of AFP observed in the DAA group compared to NDAA may be related to the more aggressive form of HCC and can explain faster relapse.

The use of DAA treatment is a new factor with an unknown impact on the tumor. One of the current hypotheses of Tatsumi et al. indicates involvement of immunological mechanisms. Dysregulation of the NK cell population in the liver caused by $C$ virus was revealed. The immediate elimination of the virus and pathogenic antigens caused by DAAs is suspected to inflict the conversion of NK cells. That could lead to the loss of immunological surveillance and accelerate the neoplasm relapse [21].

Due to the need for understandable international communication especially in oncology we also decided to present the outcome of the trial as the oneyear RFS rate using criteria proposed by ASCO - the STEEP System. STEEP defines RFS as the time from remission to any recurrence or any death [18]. Oneyear lasting RFS achieved by less than half of DAA patients $(47.3 \%)$ vs. $75 \%$ of the NDAA group $(p<0.05)$.

It is important to perform further observations of DAAs' effects on the group of patients with history of HCC surgical treatment.

\section{Conclusions}

Contemporary HCV treatment is highly effective especially in compensated cirrhosis. The trial shows that use of DAA therapy in patients with a history of HCC may result in significantly accelerated relapse of the disease and a lower one-year RFS rate, which leads to worse prognosis for this group of patients. However, the numbers in the study are too small to generalize the conclusions. Further observation with a longer follow-up and larger patient group is needed. 
Considering these facts, we suggest carefully deliberating indications to introduce the DAAs and increase the intensity of long-term post-operative care (examination with CT-scan/MRI, Duplex-Doppler USG and AFP included) in the group of patients with HCC.

\section{Disclosure}

Authors report no conflict of interest.

\section{References}

1. Thein HH, Yi Q, Dore GJ, et al. Estimation of stage-specific fibrosis progression rates in chronic hepatitis $\mathrm{C}$ virus infection: a meta-analysis and meta-regression. Hepatology 2008; 48: 418-431.

2. Seeff LB. Natural history of chronic hepatitis C. Hepatology 2002; 36 (5 Suppl 1): S35-46

3. Lok AS, Seeff LB, Morgan TR, et al. Incidence of hepatocellular carcinoma and associated risk factors in hepatitis C-related advanced liver disease. Gastroenterology 2009; 136: 138-148.

4. Sangiovanni A, Del Ninno E, Fasani P, et al. Increased survival of cirrhotic patients with a hepatocellular carcinoma detected during surveillance. Gastroenterology 2004; 126: 1005-1014.

5. Panasiuk A, Simon K, Krzmieniecki K, et al. Epidemiology of hepatocellular carcinoma in Poland (LIVER project). Clin Exp Hepatol 2016; 2: 46.

6. Fried MW, Shiffman ML, Reddy KR, et al. Peginterferon alfa-2a plus ribavirin for chronic hepatitis $C$ virus infection. N Engl J Med 2002; 347: 975-982.

7. von Wagner M, Huber M, Berg T, et al. Peginterferon-alpha-2a $(40 \mathrm{KD})$ and ribavirin for 16 or 24 weeks in patients with genotype 2 or 3 chronic hepatitis C. Gastroenterology 2005; 129: 522-527.

8. Morgan RL, Baack B, Smith BD, et al. Eradication of hepatitis C virus infection and the development of hepatocellular carcinoma: a meta-analysis of observational studies. Ann Intern Med 2013; 158 (5 Pt 1): 329-337.

9. Veldt BJ, Heathcote EJ, Wedemeyer $\mathrm{H}$, et al. Sustained virologic response and clinical outcomes in patients with chronic hepatitis $C$ and advanced fibrosis. Ann Intern Med 2007; 147: 677-684.

10. Kobayashi M, Suzuki F, Fujiyama S, et al. Sustained virologic response by direct antiviral agents reduces the incidence of hepatocellular carcinoma in patients with HCV infection. J Med Virol 2017; 89: 476-483.

11. Lawitz E, Makara M, Akarca US, et al. Efficacy and Safety of Ombitasvir, Paritaprevir, and Ritonavir in an Open-Label Study of Patients With Genotype 1b Chronic Hepatitis C Virus Infection With and Without Cirrhosis. Gastroenterology 2015; 149: 971-980.e1.

12. Charlton M, Everson GT, Flamm SL, et al. Ledipasvir and Sofosbuvir Plus Ribavirin for Treatment of HCV Infection in Patients With Advanced Liver Disease. Gastroenterology 2015; 149: 649-659.

13. Poordad F, Schiff ER, Vierling JM, et al. Daclatasvir with sofosbuvir and ribavirin for hepatitis $\mathrm{C}$ virus infection with advanced cirrhosis or post-liver transplantation recurrence. Hepatology 2016; 63: 1493-1505.

14. Attar BM, Van Thiel DH. Hepatitis C virus: A time for decisions. Who should be treated and when? World J Gastrointest Pharmacol Ther 2016; 7: 33-40.

15. Reig M, Mariño Z, Perelló C, et al. Unexpected high rate of early tumor recurrence in patients with HCV-related HCC undergoing interferon-free therapy. J Hepatol 2016; 65: 719-726.
16. Conti F, Buonfiglioli F, Scuteri A, et al. Early occurrence and recurrence of hepatocellular carcinoma in $\mathrm{HCV}$-related cirrhosis treated with direct-acting antivirals. J Hepatol 2016; 65: 727-733.

17. Yi HM, Zhang W, Ai X, et al. Radiofrequency ablation versus surgical resection for the treatment of hepatocellular carcinoma conforming to the Milan criteria: systemic review and meta-analysis. Int J Clin Exp Med 2014; 7: 3150-3163.

18. Hudis CA, Barlow WE, Costantino JP, et al. Proposal for standardized definitions for efficacy end points in adjuvant breast cancer trials: the STEEP system. J Clin Oncol 2007; 25: 21272132 .

19. ANRS collaborative study group on hepatocellular carcinoma (ANRS CO22 HEPATHER, CO12 CirVir and CO23 CUPILT cohorts). Lack of evidence of an effect of direct-acting antivirals on the recurrence of hepatocellular carcinoma: Data from three ANRS cohorts. J Hepatol 2016; 65: 734-740.

20. Di Constanzo G, Di Costanzo GG, Zamparelli MS, et al. Hepatocellular carcinoma recurrence rate in $\mathrm{HCV}$ infected patients treated with direct antiviral agents. A single center experience. J Hepatol 2017; 66 Suppl: S717.

21. Tatsumi T, Takehara T. Impact of natural killer cells on chronic hepatitis $C$ and hepatocellular carcinoma. Hepatol Res 2016; 46: 416-422. 